\title{
REPORT
}

\section{A Report on the National Workshop on "Strengthening National Capacity to Meet the Enhanced Transparency Framework of the Paris Agreement in Bangladesh"}

\author{
Md. Arfanuzzaman ${ }^{*}$ and Md. Shaheduzzaman ${ }^{* *}$
}

One of the key outcomes of the Paris Agreement was the establishment of an Enhanced Transparency Framework (ETF) for tracking and reporting the progress of countries on existing and future climate action commitments, with built-in flexibility for non-Annex I Parties. The Capacity-building Initiative for Transparency (CBIT) was created at the request of non-Annex 1 Parties to strengthen their institutional and technical capacities to meet the ETF requirements defined in Article 13 of the Paris Agreement. Till 2019, a total of 58 projects were approved by the Global Environment Facility (GEF) Secretariat including the Bangladesh project. The major objective of Bangladesh's CBIT project is to strengthen institutional and human capacities in Bangladesh to meet the requirements of the ETF and to track progress against priority mitigation and adaptation actions identified in the Nationally Determined Contributions (NDC), focusing on the agriculture, forestry, and other land use (AFOLU); energy; industrial processes and product use (IPPU); and waste sectors.

\footnotetext{
* Climate Change MRV Expert, Food and Agriculture Organization of the United Nations (FAO), House \# 37; Road \#08, Dhanmondi R/A, Dhaka-1205, Bangladesh; thisisarfan@gmail.com.

** Senior Environmental Advisor, Food and Agriculture Organization of the United Nations (FAO), House \# 37; Road \#08, Dhanmondi R/A, Dhaka-1205, Bangladesh; md.shaheduzzaman@fao.org.

The views expressed in this work are those of the authors and do not necessarily represent those of the Food and Agriculture Organization (FAO) of the United Nations.

Copyright (C) Arfanuzzaman and Shaheduzzaman 2021. Released under Creative Commons Attribution-NonCommercial 4.0 International licence (CC BY-NC 4.0) by the author.

Published by Indian Society for Ecological Economics (INSEE), c/o Institute of Economic Growth, University Enclave, North Campus, Delhi 110007.
}

ISSN: 2581-6152 (print); 2581-6101 (web).

DOI: https://doi.org/10.37773/ees.v4i1.299 
To meet its ETF requirements, Bangladesh needs to report inventories of emissions sources and sinks derived from credible and transparent data sources, which will enable the Government of Bangladesh (GoB) to track progress against priority actions identified in Bangladesh's NDC. But the limited institutional capacity for sectoral greenhouse gas (GHG) data monitoring and reporting, absence of metadata and quality-control protocols, and inadequate data sharing / archiving frameworks and policies obstruct Bangladesh's effort to meet the ETF requirements, as noted in its Third National Communication to the United Nations Framework Convention on Climate Change (UNFCCC). In this context, the Ministry of Environment Forest and Climate Change (MoEFCC), with technical support from the Food and Agriculture Organization (FAO) of the United Nations, organized a national workshop on 12 March 2020 at Dhaka under a GEF-supported CBIT project with a view to i) strengthen stakeholders' awareness of ETF requirements; ii) discuss sectoral GHG data collection and sharing arrangements; iii) conduct gap assessment for the monitoring of NDC priority adaptation and mitigation activities. A total of 83 participants from different ministries and departments, United Nations agencies, development partners, universities, think tanks, and non-government organizations as well as national and international environmental experts participated in the workshop. Of the 83 participants, $25 \%$ were female and $75 \%$ were male. The workshop solicited inputs from different stakeholders on past/ongoing projects and activities related to GHG emissions and adaptation, existing procedures, and institutional mechanisms for mitigation and adaptation-related data collection and sharing.

In the inaugural session, Mirza Shawkat Ali, Director (Climate Change \& International Convention) of the Department of Environment (DoE), MoEFCC, specified that as a signatory to the Paris Agreement, Bangladesh is committed to using specified protocols for monitoring, reporting, and verification (MRV) and tracking progress against the NDCs. However, Bangladesh has critical data gaps in the AFOLU and waste sectors as well as the energy and IPPU sectors, and this is where the CBIT project will play a crucial role in establishing an interoperable, transparent, and verifiable data collection, analysis, reporting, and archiving system.

Then, Mathieu Van Rijn, Forestry Officer, FAO Regional Office for AsiaPacific, gave a presentation on "ETF \& FAO-led CBIT Projects in Asia and the Pacific". He mentioned that the AFOLU sector is the most frequently included sector in developing countries' NDCs. He articulated that the key guiding principle of the adopted modalities, procedures, and guidelines (MPGs) for the transparency framework in COP 24 is to (i) maintain frequency and quality of reporting, and (ii) improve reporting and transparency over time. While describing the initial experiences and lessons 
from other CBIT projects, he mentioned that Papua New Guinea has prepared an AFOLU Action Plan and Mongolia has established an NDC Forum that addresses the need for well-structured institutional arrangements and transparent GHG data collection and reporting. While speaking as a Special Guest, Robert D Simpson, FAO Representative in Bangladesh, stressed the importance of a well-designed policy for achieving the Sustainable Development Goals (SDGs), which must include a rigorous effort to address environmental and climate change issues. He stated that the global response to climate change today will determine how we feed future generations tomorrow. Agriculture, including forestry, crops, fisheries, and livestock production, generates around a fifth of the world's GHG emissions. This must be significantly reduced by 2030 to achieve the goal of limiting global warming to 2 degree Celsius.

A. Shamim Al Razi, Additional Secretary (Development), MoEFCC, mentioned that strengthening individual and institutional capacities in Bangladesh is essential to meet the ETF requirements. He noted that Bangladesh has a huge data deficit with regards the environment and natural resource sector, where special attention is required to enhance environmental governance. Ziaul Hasan, Secretary, MoEFCC, in his remarks as Chief Guest, emphasized the need to strengthen the technical capacities of Bangladesh to meet the ETF requirements of the Paris Agreement. He urged the project administration to set up a common and user-friendly platform for GHG data sharing and coordination to comply with the TCCCA (transparent, consistent, comparable, complete, and accurate) reporting principles. Ziaul Hasan assured that the MoEFCC will take the lead in addressing the environmental issues of the country.

Following the inaugural session, nearly 50 participants from different organizations engaged in a group discussion. In general, there was a high degree of support from the participants for a formal memorandum of understanding $(\mathrm{MoU})$ between the DoE and the respective entities on GHG data collection. Participants also noted that universities, which conduct research on emissions factors and activities for various sectors, are not always linked to line ministries and departments. DoE / MoEFCC could form a panel of experts to review of the Biennial Update Report (BUR) and Biennial Transparency Report (BTR) and recognize them officially for their contribution in the reports. Group discussants also expressed interest in technical capacity-building workshops, since many data providers are not entirely familiar with UNFCCC or ETF / MRV related requirements. Discussants noted that the waste sector will require focused capacity-building efforts since city corporations and municipalities often lack record-keeping, despite waste management policies and projects. 
In their evaluations of the workshop, most of the participants recommended the development of a unified data collection template (including meta-data standards) and indicated that this should be followed by training. Participants also proposed the formation of a technical working group to check the accuracy and quality of the GHG emissions data of Bangladesh. Participants urged that to close capacity gaps, Bangladesh needs to have an intensive focus on the following aspects:

(i) institutional arrangements to support the transition to ETF;

(ii) technical capacities for mitigation-related MRV; and

(iii) capacities for adaptation-related monitoring and reporting.

Finally, in addition to the efforts towards meeting the ETF requirements, Bangladesh as well as other developing countries need to pay sufficient attention to environmental equality enhancement and green economy advancement. To attain this, strong political commitment, as well as institutional arrangements and capacity-building, a robust NDC implementation strategy, private-sector engagement, technological innovation, and law and enforcement, need to be strengthened. Nevertheless, there is no alternative for awareness-raising on transparent GHG emission measurement, monitoring, and reporting, and effective mitigation action.

\section{ACKNOWLEDGMENT}

The authors are grateful to the editor of the journal and the anonymous reviewers for providing useful comments and suggestion to further improve the paper. 